\title{
A New Class of Analytic Functions Defined by Using Salagean Operator
}

\author{
R. M. El-Ashwah, ${ }^{1}$ M. K. Aouf, ${ }^{2}$ A. A. M. Hassan, ${ }^{3}$ and A. H. Hassan ${ }^{3}$ \\ ${ }^{1}$ Department of Mathematics, Faculty of Science, Damietta University, New Damietta 34517, Egypt \\ ${ }^{2}$ Department of Mathematics, Faculty of Science, Mansoura University, Mansoura 33516, Egypt \\ ${ }^{3}$ Department of Mathematics, Faculty of Science, Zagazig University, Zagazig 44519, Egypt
}

Correspondence should be addressed to A. H. Hassan; alaahassan1986@yahoo.com

Received 14 August 2012; Revised 6 November 2012; Accepted 12 November 2012

Academic Editor: Yaozhong Hu

Copyright (c) 2013 R. M. El-Ashwah et al. This is an open access article distributed under the Creative Commons Attribution License, which permits unrestricted use, distribution, and reproduction in any medium, provided the original work is properly cited.

We derive some results for a new class of analytic functions defined by using Salagean operator. We give some properties of functions in this class and obtain numerous sharp results including for example, coefficient estimates, distortion theorem, radii of star-likeness, convexity, close-to-convexity, extreme points, integral means inequalities, and partial sums of functions belonging to this class. Finally, we give an application involving certain fractional calculus operators that are also considered.

\section{Introduction}

Let $\mathscr{A}$ denote the class of functions of the form

$$
f(z)=z+\sum_{k=2}^{\infty} a_{k} z^{k}
$$

that are analytic and univalent in the open unit $\operatorname{disc} U=\{z \epsilon$ $\mathbb{C}:|z|<1\}$.

For $f(z) \in \mathscr{A}$, Salagean [1] introduced the following differential operator: $D^{0} f(z)=f(z), D^{1} f(z)=z f^{\prime}(z), \ldots$, $D^{n} f(z)=D\left(D^{n-1} f(z)\right)(n \in N=\{1,2, \ldots\})$.

We note that

$$
D^{n} f(z)=z+\sum_{k=2}^{\infty} k^{n} a_{k} z^{k} \quad\left(n \in \mathbb{N}_{0}=\mathbb{N} \cup\{0\}\right) .
$$

Definition 1 (subordination principle). For two functions $f$ and $g$, analytic in $U$, we say that the function $f(z)$ is subordinate to $g(z)$ in $U$ and write $f(z) \prec g(z)$, if there exists a Schwarz function $w(z)$, which (by definition) is analytic in $U$ with $w(0)=0$ and $|w(z)|<1$, such that $f(z)=$ $g(w(z))(z \in U)$. Indeed it is known that

$$
f(z) \prec g(z) \Longrightarrow f(0)=g(0), \quad f(U) \subset g(U) .
$$

Furthermore, if the function $g$ is univalent in $U$, then we have the following equivalence [2, page 4$]$ :

$$
f(z) \prec g(z) \Longleftrightarrow f(0)=g(0), \quad f(U) \subset g(U)
$$

Definition 2 (see [3]). Let $U_{m, n}(\beta, A, B)$ denote the subclass of $\mathscr{A}$ consisting of functions $f(z)$ of the form (1) and satisfy the following subordination:

$$
\begin{array}{r}
\frac{D^{m} f(z)}{D^{n} f(z)}-\beta\left|\frac{D^{m} f(z)}{D^{n} f(z)}-1\right|<\frac{1+A z}{1+B z}, \\
(-1 \leq B<A \leq 1 ;-1 \leq B<0 ; \beta \geq 0 ; \\
\left.m \in \mathbb{N} ; n \in \mathbb{N}_{0}, m>n ; z \in U\right) .
\end{array}
$$

Specializing the parameters $A, B, \beta, m$, and $n$, we obtain the following subclasses studied by various authors: 
(i)

$$
\begin{aligned}
U_{m, n} & (\beta, 1-2 \alpha,-1) \\
= & N_{m, n}(\alpha, \beta) \\
= & \left\{f \in \mathscr{A}: \operatorname{Re}\left\{\frac{D^{m} f(z)}{D^{n} f(z)}-\alpha\right\}>\beta\left|\frac{D^{m} f(z)}{D^{n} f(z)}-1\right|\right. \\
& \left.\quad\left(0 \leq \alpha<1 ; \beta \geq 0 ; m \in \mathbb{N} ; n \in \mathbb{N}_{0} ; m>n ; z \in U\right)\right\},
\end{aligned}
$$

(see Eker and Owa [4]);

(ii)

$$
\begin{aligned}
& U_{1,0}(\beta, 1-2 \alpha,-1) \\
& =U S(\alpha, \beta) \\
& =\left\{f \in \mathscr{A}: \operatorname{Re}\left\{\frac{z f^{\prime}(z)}{f(z)}-\alpha\right\}>\beta\left|\frac{z f^{\prime}(z)}{f(z)}-1\right|\right. \\
& U_{2,1}(\beta, 1-2 \alpha,-1) \\
& =U K(\alpha, \beta) \quad(0 \leq \alpha<1 ; \beta \geq 0 ; z \in U)\}, \\
& =\left\{f \in \mathscr{A}: \operatorname{Re}\left\{1+\frac{z f^{\prime \prime}(z)}{f^{\prime}(z)}-\alpha\right\}>\beta\left|\frac{z f^{\prime \prime}(z)}{f^{\prime}(z)}\right|\right. \\
& \quad(0 \leq \alpha<1 ; \beta \geq 0 ; z \in U)\},
\end{aligned}
$$

(see Shams et al. $[5,6])$;

(iii)

$$
\begin{aligned}
& U_{1,0}(0, A, B) \\
& =S^{*}(A, B) \\
& =\left\{f \in \mathscr{A}: \frac{z f^{\prime}(z)}{f(z)} \prec \frac{1+A z}{1+B z}\right. \\
& \quad(-1 \leq B<A \leq 1 ; z \in U)\}, \\
& U_{2,1}(0, A, B) \\
& =K(A, B) \\
& =\left\{f \in \mathscr{A}: 1+\frac{z f^{\prime \prime}(z)}{f^{\prime}(z)} \prec \frac{1+A z}{1+B z}\right. \\
& (-1 \leq B<A \leq 1 ; z \in U)\},
\end{aligned}
$$

(see Janowski [7] and Padmanabhan and Ganesan [8]).
Also we note that

$$
\begin{aligned}
U_{m, n}( & 0, A, B) \\
= & U(m, n ; A, B) \\
= & \left\{f(z) \in \mathscr{A}: \frac{D^{m} f(z)}{D^{n} f(z)} \prec \frac{1+A z}{1+B z}\right. \\
& \left.\quad\left(-1 \leq B<A \leq 1 ; m \in \mathbb{N} ; n \in \mathbb{N}_{0} ; m>n ; z \in U\right)\right\} .
\end{aligned}
$$

Let $T$ denote the subclass of functions of $\mathscr{A}$ of the form

$$
f(z)=z-\sum_{k=2}^{\infty} a_{k} z^{k}, \quad a_{k} \geq 0 .
$$

Further, we define the class $T S_{\gamma}(f, g ; \alpha, \beta)$ by

$$
T U_{m, n}(\beta, A, B)=U_{m, n}(\beta, A, B) \cap T .
$$

For suitable choices of the parameters $A, B, \beta, m$, and $n$, we can get various known or new subclasses of $T$. For example, we have the following:

(i) $T U_{n+1, n}(\beta, 1-2 \alpha,-1)=T S(n, \alpha, \beta)(0 \leq \alpha<1, \beta \geq$ $0, n \in \mathbb{N}_{0}$ ) (see Rosy and Murugusundaramoorthy [9] and Aouf [10]);

(ii) $T U_{1,0}(1,1-2 \alpha,-1)=S_{p} T(\alpha)$ and $T U_{2,1}(1,1-$ $2 \alpha,-1)=U C T(\alpha)(0 \leq \alpha<1)$ (see Bharati et al. $[11])$

(iii) $T U_{1,0}(0,1-2 \alpha,-1)=T^{*}(\alpha)$ and $T U_{2,1}(0,1-$ $2 \alpha,-1)=C(\alpha)(0 \leq \alpha<1)$ (see Silverman [12]).

\section{Coefficient Estimates}

Unless otherwise mentioned, we assume in the reminder of this paper that $-1 \leq B<A \leq 1,-1 \leq B<0, \beta \geq 0, m \in$ $\mathbb{N}, n \in \mathbb{N}_{0}, m>n$ and $z \in U$.

Now, we will need the following lemma which gives a sufficient condition for functions belonging to the class $U_{m, n}(\beta, A, B)$.

Lemma 3 (see [13]). A function $f(z)$ of the form (1) is in the class $U_{m, n}(\beta, A, B)$ if

$$
\sum_{k=2}^{\infty}\left[(1+\beta(1+|B|))\left(k^{m}-k^{n}\right)+\left|B k^{m}-A k^{n}\right|\right]\left|a_{k}\right| \leq A-B .
$$

In Theorem 4, it is shown that the condition (12) is also necessary for functions $f(z)$ of the form (10) to be in the class $T U_{m, n}(\beta, A, B)$.

Theorem 4. Let $f(z) \in T$. Then $f(z) \in T U_{m, n}(\beta, A, B)$ if and only if

$$
\sum_{k=2}^{\infty}\left[(1+\beta(1+|B|))\left(k^{m}-k^{n}\right)+\left|B k^{m}-A k^{n}\right|\right] a_{k} \leq A-B .
$$


Proof. In view of Lemma 3, we only need to prove the only if part of Theorem 4 . Since $T U_{m, n}(\beta, A, B) \subset U_{m, n}(\beta, A, B)$, for functions $f(z) \in T U_{m, n}(\beta, A, B)$, we can write

$$
\begin{aligned}
& \left|\frac{p(z)-1}{A-B p(z)}\right|<1, \\
& \quad \text { where } p(z)=\frac{D^{m} f(z)}{D^{n} f(z)}-\beta\left|\frac{D^{m} f(z)}{D^{n} f(z)}-1\right| .
\end{aligned}
$$

then

$$
\begin{gathered}
\mid\left(\sum_{k=2}^{\infty}\left(k^{m}-k^{n}\right) a_{k} z^{k}+\beta e^{i \theta}\left|\sum_{k=2}^{\infty}\left(k^{m}-k^{n}\right) a_{k} z^{k}\right|\right) \\
\times\left((A-B) z+\sum_{k=2}^{\infty}\left(B k^{m}-A k^{n}\right) a_{k} z^{k}\right. \\
\left.+B \beta e^{i \theta}\left|\sum_{k=2}^{\infty}\left(k^{m}-k^{n}\right) a_{k} z^{k}\right|\right)^{-1} \mid<1 .
\end{gathered}
$$

$$
\begin{gathered}
\times\left((A-B) z+\sum_{k=2}^{\infty}\left(B k^{m}-A k^{n}\right) a_{k} z^{k}\right. \\
\left.\left.+B \beta e^{i \theta}\left|\sum_{k=2}^{\infty}\left(k^{m}-k^{n}\right) a_{k} z^{k}\right|\right)^{-1}\right\}<1 .
\end{gathered}
$$

Now choosing $z$ to be real and letting $z \rightarrow 1^{-}$, we obtain

$$
\sum_{k=2}^{\infty}\left[(1+\beta(1-B))\left(k^{m}-k^{n}\right)-\left(B k^{m}-A k^{n}\right)\right] a_{k} \leq A-B .
$$

Or, equivalently

$$
\sum_{k=2}^{\infty}\left[(1+\beta(1+|B|))\left(k^{m}-k^{n}\right)+\left|B k^{m}-A k^{n}\right|\right] a_{k} \leq A-B .
$$

This completes the proof of Theorem 4 .

Remark 5. (i) The result obtained by Theorem 4 corrects the result obtained by Li and Tang [3, Theorem 1].

(ii) Putting $A=1-2 \alpha(0 \leq \alpha<1)$ and $B=-1$ in Theorem 4, we correct the result obtained by Eker and Owa [4, Theorem 2.1].

(iii) Putting $A=1-2 \alpha(0 \leq \alpha<1), B=-1$, and $m=$ $n+1\left(n \in \mathbb{N}_{0}\right)$ in Theorem 4 , we obtain the result obtained by Rosy and Murugusudaramoorthy [9, Theorem 2].
Corollary 6. Let the function $f(z)$ be defined by (10) and let it be in the class $T U_{m, n}(\beta, A, B)$. Then

$$
\begin{array}{r}
a_{k} \leq \frac{A-B}{(1+\beta(1+|B|))\left(k^{m}-k^{n}\right)+\left|B k^{m}-A k^{n}\right|}, \\
(k \geq 2) .
\end{array}
$$

The result is sharp for the function

$$
f(z)=z-\frac{A-B}{(1+\beta(1+|B|))\left(k^{m}-k^{n}\right)+\left|B k^{m}-A k^{n}\right|} z^{k},
$$

\section{Distortion Theorems}

Theorem 7. Let the function $f(z)$ defined by (10) be in the class $T U_{m, n}(\beta, A, B)$. Then

$$
\begin{aligned}
|f(z)| & \geq|z|-\frac{A-B}{(1+\beta(1+|B|))\left(2^{m}-2^{n}\right)+\left|B 2^{m}-A 2^{n}\right|}|z|^{2} \\
& \leq|z|+\frac{A-B}{(1+\beta(1+|B|))\left(2^{m}-2^{n}\right)+\left|B 2^{m}-A 2^{n}\right|}|z|^{2} .
\end{aligned}
$$

The result is sharp.

Proof. In view of Theorem 4, since

$$
\Phi(k)=(1+\beta(1+|B|))\left(k^{m}-k^{n}\right)+\left|B k^{m}-A k^{n}\right|
$$

is an increasing function of $k(k \geq 2)$, we have

$$
\Phi(2) \sum_{k=2}^{\infty}\left|a_{k}\right| \leq \sum_{k=2}^{\infty} \Phi(k)\left|a_{k}\right| \leq A-B
$$

that is

$$
\sum_{k=2}^{\infty}\left|a_{k}\right| \leq \frac{A-B}{\Phi(2)}
$$

Thus we have

$$
\begin{gathered}
|f(z)| \leq|z|+|z|^{2} \sum_{k=2}^{\infty}\left|a_{k}\right|, \\
|f(z)| \leq|z|+\frac{A-B}{(1+\beta(1+|B|))\left(2^{m}-2^{n}\right)+\left|B 2^{m}-A 2^{n}\right|}|z|^{2} .
\end{gathered}
$$

Similarly, we get

$$
\begin{aligned}
|f(z)| & \geq|z|-\sum_{k=2}^{\infty}\left|a_{k}\right||z|^{k} \geq|z|-|z|^{2} \sum_{k=2}^{\infty}\left|a_{k}\right| \\
& \geq|z|-\frac{A-B}{(1+\beta(1+|B|))\left(2^{m}-2^{n}\right)+\left|B 2^{m}-A 2^{n}\right|}|z|^{2} .
\end{aligned}
$$


Finally the result is sharp for the function

$$
f(z)=z-\frac{A-B}{(1+\beta(1+|B|))\left(2^{m}-2^{n}\right)+\left|B 2^{m}-A 2^{n}\right|} z^{2},
$$

at $z=r$ and $z=r e^{i(2 k+1) \pi}(k \in \mathbb{Z}=\{\ldots,-2,-1,0,1,2, \ldots\})$. This completes the proof of Theorem 7 .

Theorem 8. Let the function $f(z)$ defined by (10) be in the class $T U_{m, n}(\beta, A, B)$. Then

$$
\begin{aligned}
\left|f^{\prime}(z)\right| & \geq 1-\frac{2(A-B)}{(1+\beta(1+|B|))\left(2^{m}-2^{n}\right)+\left|B 2^{m}-A 2^{n}\right|}|z| \\
& \leq 1+\frac{2(A-B)}{(1+\beta(1+|B|))\left(2^{m}-2^{n}\right)+\left|B 2^{m}-A 2^{n}\right|}|z| .
\end{aligned}
$$

The result is sharp.

Proof. Similarly $\Phi(k) / k$ is an increasing function of $k(k \geq 2)$, in view of Theorem 4 , we have

$$
\frac{\Phi(2)}{2} \sum_{k=2}^{\infty} k\left|a_{k}\right| \leq \sum_{k=2}^{\infty} \frac{\Phi(k)}{k} k\left|a_{k}\right|=\sum_{k=2}^{\infty} \Phi(k)\left|a_{k}\right| \leq A-B,
$$

that is

$$
\sum_{k=2}^{\infty} k\left|a_{k}\right| \leq \frac{2(A-B)}{\Phi(2)}
$$

Thus we have

$$
\begin{aligned}
\left|f^{\prime}(z)\right| & \leq 1+|z| \sum_{k=2}^{\infty} k\left|a_{k}\right| \\
& \leq 1+\frac{2(A-B)}{(1+\beta(1+|B|))\left(2^{m}-2^{n}\right)+\left|B 2^{m}-A 2^{n}\right|}|z| .
\end{aligned}
$$

Similarly

$$
\begin{aligned}
\left|f^{\prime}(z)\right| & \geq 1-|z| \sum_{k=2}^{\infty} k\left|a_{k}\right| \\
& \geq 1-\frac{2(A-B)}{(1+\beta(1+|B|))\left(2^{m}-2^{n}\right)+\left|B 2^{m}-A 2^{n}\right|}|z| .
\end{aligned}
$$

Finally, we can see that the assertions of Theorem 8 are sharp for the function $f(z)$ defined by (27). This completes the proof of Theorem 8 .

\section{Radii of Starlikeness, Convexity, and Close-to-Convexity}

In this section radii of close-to-convexity, starlikeness, and convexity for functions belonging to the class $T U_{m, n}(\beta, A, B)$ are obtained.
Theorem 9. Let the function $f(z)$ defined by (10) be in the class $T U_{m, n}(\beta, A, B)$; then

(i) $f(z)$ is starlike of order $\varphi(0 \leq \varphi<1)$ in $|z|<r_{1}$, where

$$
\begin{aligned}
r_{1}=\inf _{k \geq 2}\left\{\frac{(1+\beta(1+|B|))\left(k^{m}-k^{n}\right)+\left|B k^{m}-A k^{n}\right|}{A-B}\right. \\
\left.\times\left(\frac{1-\varphi}{k-\varphi}\right)\right\}^{1 /(k-1)}
\end{aligned}
$$

(ii) $f(z)$ is convex of order $\varphi(0 \leq \varphi<1)$ in $|z|<r_{2}$, where

$$
r_{2}=\inf _{k \geq 2}\left\{\frac{(1+\beta(1+|B|))\left(k^{m}-k^{n}\right)+\left|B k^{m}-A k^{n}\right|}{A-B}\right.
$$$$
\left.\times \frac{(1-\varphi)}{k(k-\varphi)}\right\}^{1 /(k-1)}
$$

(iii) $f(z)$ is close-to-convex of $\operatorname{order} \varphi(0 \leq \varphi<1)$ in $|z|<$ $r_{3}$, where

$$
\begin{aligned}
r_{3}=\inf _{k \geq 2}\{ & \frac{(1+\beta(1+|B|))\left(k^{m}-k^{n}\right)+\left|B k^{m}-A k^{n}\right|}{A-B} \\
& \left.\times\left(\frac{1-\varphi}{k}\right)\right\}^{1 /(k-1)} .
\end{aligned}
$$

Each of these results is sharp for the function $f(z)$ given by (20). Proof. It is sufficient to show that

$$
\left|\frac{z f^{\prime}(z)}{f(z)}-1\right| \leq 1-\varphi, \quad \text { for }|z|<r_{1},
$$

where $r_{1}$ is given by (33). Indeed we find from (10) that

$$
\left|\frac{z f^{\prime}(z)}{f(z)}-1\right| \leq \frac{\sum_{k=2}^{\infty}(k-1) a_{k}|z|^{k-1}}{1-\sum_{k=2}^{\infty} a_{k}|z|^{k-1}} .
$$

Thus we have

$$
\left|\frac{z f^{\prime}(z)}{f(z)}-1\right| \leq 1-\varphi
$$

if and only if

$$
\sum_{k=2}^{\infty} \frac{(k-\varphi) a_{k}|z|^{k-1}}{(1-\varphi)} \leq 1
$$

But, by Theorem 4, (39) will be true if

$$
\frac{(k-\varphi)|z|^{k-1}}{(1-\varphi)} \leq \frac{(1+\beta(1+|B|))\left(k^{m}-k^{n}\right)+\left|B k^{m}-A k^{n}\right|}{A-B},
$$


that is, if

$$
\begin{gathered}
|z| \leq\left\{\frac{(1+\beta(1+|B|))\left(k^{m}-k^{n}\right)+\left|B k^{m}-A k^{n}\right|}{A-B}\right. \\
\left.\quad \times\left(\frac{1-\varphi}{k-\varphi}\right)\right\}^{1 /(k-1)} \quad(k \geq 2) .
\end{gathered}
$$

Or

$$
\begin{gathered}
r_{1}=\inf _{k \geq 2}\left\{\frac{(1+\beta(1+|B|))\left(k^{m}-k^{n}\right)+\left|B k^{m}-A k^{n}\right|}{A-B}\right. \\
\left.\quad \times\left(\frac{1-\varphi}{k-\varphi}\right)\right\}^{1 /(k-1)} .
\end{gathered}
$$

This completes the proof of (33).

To prove (34) and (35) it is sufficient to show that

$$
\begin{gathered}
\left|1+\frac{z f^{\prime \prime}(z)}{f^{\prime}(z)}-1\right| \leq 1-\varphi \quad\left(|z|<r_{2} ; 0 \leq \varphi<1\right), \\
\left|f^{\prime}(z)-1\right| \leq 1-\varphi \quad\left(|z|<r_{3} ; 0 \leq \varphi<1\right),
\end{gathered}
$$

respectively.

\section{Extreme Points}

Theorem 10. Let

$$
\begin{gathered}
f_{1}(z)=z, \\
f_{k}(z)=z-\frac{A-B}{(1+\beta(1+|B|))\left(k^{m}-k^{n}\right)+\left|B k^{m}-A k^{n}\right|} z^{k}, \\
(k=2,3, \ldots) .
\end{gathered}
$$

Then $f(z) \in T U_{m, n}(\beta, A, B)$ if and only if it can be expressed in the following form:

$$
f(z)=\sum_{k=1}^{\infty} \eta_{k} f_{k}(z)
$$

where

$$
\eta_{k} \geq 0, \quad \sum_{k=1}^{\infty} \eta_{k}=1 .
$$

Proof. Suppose that

$$
\begin{aligned}
f(z) & =\sum_{k=1}^{\infty} \eta_{k} f_{k}(z) \\
& =z-\sum_{k=2}^{\infty} \eta_{k} \frac{A-B}{(1+\beta(1+|B|))\left(k^{m}-k^{n}\right)+\left|B k^{m}-A k^{n}\right|} z^{k} .
\end{aligned}
$$

Then, from Theorem 4, we have

$$
\begin{aligned}
\sum_{k=2}^{\infty}[ & \left\{(1+\beta(1+|B|))\left(k^{m}-k^{n}\right)+\left|B k^{m}-A k^{n}\right|\right\} \\
& \left.\cdot \frac{A-B}{(1+\beta(1+|B|))\left(k^{m}-k^{n}\right)+\left|B k^{m}-A k^{n}\right|} \eta_{k}\right] \\
= & (A-B) \sum_{k=2}^{\infty} \eta_{k}=(A-B)\left(1-\eta_{1}\right) \leq A-B .
\end{aligned}
$$

Thus, in view of Theorem 4, we find that $f(z) \in$ $T U_{m, n}(\beta, A, B)$.

Conversely, let us suppose that $f(z) \in T U_{m, n}(\beta, A, B)$, then, since

$$
\begin{array}{r}
a_{k} \leq \frac{A-B}{(1+\beta(1+|B|))\left(k^{m}-k^{n}\right)+\left|B k^{m}-A k^{n}\right|}, \\
(k=2,3, \ldots) .
\end{array}
$$

Set

$$
\begin{gathered}
\eta_{k}=\frac{(1+\beta(1+|B|))\left(k^{m}-k^{n}\right)+\left|B k^{m}-A k^{n}\right|}{A-B} a_{k}, \\
(k=2,3, \ldots), \\
\eta_{1}=1-\sum_{k=2}^{\infty} \eta_{k} .
\end{gathered}
$$

Thus clearly, we have

$$
f(z)=\sum_{k=1}^{\infty} \eta_{k} f_{k}(z) .
$$

This completes the proof of Theorem 10 .

Corollary 11. The extreme points of the class $T U_{m, n}(\beta, A, B)$ are given by

$$
\begin{gathered}
f_{1}(z)=z, \\
f_{k}(z)=z-\frac{A-B}{(1+\beta(1+|B|))\left(k^{m}-k^{n}\right)+\left|B k^{m}-A k^{n}\right|} z^{k} \\
(k=2,3, \ldots) .
\end{gathered}
$$

\section{Integral Means Inequalities}

In 1925, Littlewood [14] proved the following subordination lemma.

Lemma 12. If the functions $f$ and $g$ are analytic in $U$ with

$$
f(z) \prec g(z) \quad(z \in U),
$$

then for $p>0$ and $z=r e^{i \theta}(0<r<1)$,

$$
\int_{0}^{2 \pi}|f(z)|^{p} d \theta \leq \int_{0}^{2 \pi}|g(z)|^{p} d \theta .
$$

We now make use of Lemma 12 to prove Theorem 13. 
Theorem 13. Suppose that $f(z) \in T U_{m, n}(\beta, A, B), p>$ $0,-1 \leq B<A \leq 1, \beta>0, m \in \mathbb{N}, n \in \mathbb{N}_{0}, m>n$, and $f_{2}(z)$ is defined by

$$
f_{2}(z)=z-\frac{A-B}{(1+\beta(1+|B|))\left(2^{m}-2^{n}\right)+\left|B 2^{m}-A 2^{n}\right|} z^{2} .
$$

Then for $z=r e^{i \theta}(0<r<1)$, we have

$$
\int_{0}^{2 \pi}|f(z)|^{p} d \theta \leq \int_{0}^{2 \pi}\left|f_{2}(z)\right|^{p} d \theta
$$

Proof. For $f(z)=z-\sum_{k=2}^{\infty} a_{k} z^{k}\left(a_{k} \geq 0\right)$, (55) is equivalent to prove that

$$
\begin{aligned}
& \int_{0}^{2 \pi}\left|1-\sum_{k=2}^{\infty} a_{k} z^{k-1}\right|^{p} d \theta \\
& \quad \leq \int_{0}^{2 \pi}\left|1-\frac{A-B}{(1+\beta(1+|B|))\left(2^{m}-2^{n}\right)+\left|B 2^{m}-A 2^{n}\right|} z\right|^{p} d \theta .
\end{aligned}
$$

By applying Littlewood's subordination lemma (Lemma 12), it would suffice to show that

$$
\begin{aligned}
1-\sum_{k=2}^{\infty} a_{k} z^{k-1} & \\
& \prec 1-\frac{A-B}{(1+\beta(1+|B|))\left(2^{m}-2^{n}\right)+\left|B 2^{m}-A 2^{n}\right|} z .
\end{aligned}
$$

By setting

$$
\begin{aligned}
& 1-\sum_{k=2}^{\infty} a_{k} z^{k-1} \\
& \quad=1-\frac{A-B}{(1+\beta(1+|B|))\left(2^{m}-2^{n}\right)+\left|B 2^{m}-A 2^{n}\right|} w(z)
\end{aligned}
$$

and using (13), we obtain

$$
\begin{aligned}
& |w(z)| \\
& \quad=\left|\sum_{k=2}^{\infty} \frac{(1+\beta(1+|B|))\left(2^{m}-2^{n}\right)+\left|B 2^{m}-A 2^{n}\right|}{A-B} a_{k} z^{k-1}\right| \\
& \quad \leq|z| \sum_{k=2}^{\infty} \frac{(1+\beta(1+|B|))\left(2^{m}-2^{n}\right)+\left|B 2^{m}-A 2^{n}\right|}{A-B} a_{k} \\
& \quad \leq|z| \sum_{k=2}^{\infty} \frac{(1+\beta(1+|B|))\left(k^{m}-k^{n}\right)+\left|B k^{m}-A k^{n}\right|}{A-B} a_{k}
\end{aligned}
$$$$
\leq|z|<1 \text {. }
$$

\section{Partial Sums}

In this section partial sums of functions in the class $U_{m, n}(\beta, A, B)$ are obtained, also we will obtain sharp lower bounds for the ratios of real part of $f(z)$ to $f_{n}(z)$.

Theorem 14. Define the partial sums $f_{1}(z)$ and $f_{n}(z)$ by

$$
f_{1}(z)=z, \quad f_{n}(z)=z+\sum_{k=2}^{n} a_{k} z^{k} \quad(n \in \mathbb{N} \backslash\{1\}) .
$$

Let the function $f(z) \in U_{m, n}(\beta, A, B)$ be given by (1) and let it satisfy the condition (12) and

$$
c_{k} \geq \begin{cases}1, & k=2,3, \ldots, n, \\ c_{n+1}, & k=n+1, n+2, \ldots,\end{cases}
$$

where, for convenience,

$$
c_{k}=\frac{(1+\beta(1+|B|))\left(k^{m}-k^{n}\right)+\left|B k^{m}-A k^{n}\right|}{A-B},
$$

Then

$$
\begin{gathered}
\operatorname{Re}\left\{\frac{f(z)}{f_{n}(z)}\right\}>1-\frac{1}{c_{n+1}} \quad(z \in U ; n \in \mathbb{N}), \\
\operatorname{Re}\left\{\frac{f_{n}(z)}{f(z)}\right\}>\frac{c_{n+1}}{1+c_{n+1}} .
\end{gathered}
$$

Proof. For the coefficients $c_{k}$ given by (64) it is not difficult to verify that

$$
c_{k+1}>c_{k}>1 \text {. }
$$

Therefore we have

$$
\sum_{k=2}^{n}\left|a_{k}\right|+c_{n+1} \sum_{k=n+1}^{\infty}\left|a_{k}\right| \leq \sum_{k=2}^{\infty} c_{k}\left|a_{k}\right| \leq 1 .
$$

By setting

$$
\begin{aligned}
g_{1}(z) & =c_{n+1}\left\{\frac{f(z)}{f_{n}(z)}-\left(1-\frac{1}{c_{n+1}}\right)\right\} \\
& =1+\frac{c_{n+1} \sum_{k=n+1}^{\infty} a_{k} z^{k-1}}{1+\sum_{k=2}^{n} a_{k} z^{k-1}}
\end{aligned}
$$

and applying (68), we find that

$$
\left|\frac{g_{1}(z)-1}{g_{1}(z)+1}\right|<\frac{c_{n+1} \sum_{k=n+1}^{\infty}\left|a_{k}\right|}{2-2 \sum_{k=2}^{n}\left|a_{k}\right|-c_{n+1} \sum_{k=n+1}^{\infty}\left|a_{k}\right|} .
$$

Now

$$
\left|\frac{g_{1}(z)-1}{g_{1}(z)+1}\right|<1
$$

if

$$
\sum_{k=2}^{n}\left|a_{k}\right|+c_{n+1} \sum_{k=n+1}^{\infty}\left|a_{k}\right| \leq 1 .
$$

This completes the proof of Theorem 13. 
From the condition (12), it is sufficient to show that

$$
\sum_{k=2}^{n}\left|a_{k}\right|+c_{n+1} \sum_{k=n+1}^{\infty}\left|a_{k}\right| \leq \sum_{k=2}^{\infty} c_{k}\left|a_{k}\right|
$$

which is equivalent to

$$
\sum_{k=2}^{n}\left(c_{k}-1\right)\left|a_{k}\right|+\sum_{k=n+1}^{\infty}\left(c_{k}-c_{n+1}\right)\left|a_{k}\right| \geq 0,
$$

which readily yields the assertion (65) of Theorem 14. In order to see that

$$
f(z)=z+\frac{z^{n+1}}{c_{n+1}}
$$

gives sharp result, we observe that for $z=r e^{i \pi / n}$ that $f(z) / f_{n}(z)=1+z^{n} / c_{n+1} \rightarrow 1-1 / c_{n+1}$ as $z \rightarrow 1^{-}$. Similarly, if we take

$$
\begin{aligned}
g_{2}(z) & =\left(1+c_{n+1}\right)\left\{\frac{f_{n}(z)}{f(z)}-\frac{c_{n+1}}{1+c_{n+1}}\right\} \\
& =1-\frac{\left(1+c_{n+1}\right) \sum_{k=n+1}^{\infty} a_{k} z^{k-1}}{1+\sum_{k=2}^{\infty} a_{k} z^{k-1}}
\end{aligned}
$$

and making use of (68), we can deduce that

$$
\left|\frac{g_{2}(z)-1}{g_{2}(z)+1}\right|<\frac{\left(1+c_{n+1}\right) \sum_{k=n+1}^{\infty}\left|a_{k}\right|}{2-2 \sum_{k=2}^{n}\left|a_{k}\right|-\left(1-c_{n+1}\right) \sum_{k=n+1}^{\infty}\left|a_{k}\right|}
$$

which leads us immediately to the assertion (66) of Theorem 14.

The bound in (66) is sharp for each $n \in \mathbb{N}$ with the extremal function $f(z)$ given by (75). Then the proof of Theorem 14 is completed.

\section{Distortion Theorems Involving Fractional Calculus}

In this section, we will prove several distortion theorems for functions belonging to the class $T U_{m, n}(\beta, A, B)$. Each of these theorems would involve certain operators of fractional calculus (i.e., fractional integrals and fractional derivatives), which are defined as follows (see, for details, [15-18]). For our present investigation, we recall the following definitions.

Definition 15. The fractional integral of order $\delta$ is defined, for a function $f(z)$, by

$$
D_{z}^{-\delta} f(z)=\frac{1}{\Gamma(\delta)} \int_{0}^{z} \frac{f(\zeta)}{(z-\zeta)^{1-\delta}} d \zeta \quad(\delta>0),
$$

where the function $f(z)$ is analytic in a simply connected domain of the complex $z$-plane containing the origin, and the multiplicity of $(z-\zeta)^{\delta-1}$ is removed by requiring $\log (z-\zeta)$ to be real when $z-\zeta>0$.
Definition 16. The fractional derivative of order $\delta$ is defined, for a function $f(z)$, by

$$
D_{z}^{\delta} f(z)=\frac{1}{\Gamma(1-\delta)} \frac{d}{d z} \int_{0}^{z} \frac{f(\zeta)}{(z-\zeta)^{\delta}} d \zeta \quad(0 \leq \delta<1),
$$

where the function $f(z)$ is constrained, and the multiplicity of $(z-\zeta)^{-\delta}$ is removed as in Definition 15.

Definition 17. Under the hypotheses of Definition 16, the fractional derivative of order $\delta$ is defined, for a function $f(z)$, by

$$
D_{z}^{n+\delta} f(z)=\frac{d^{n}}{d z^{n}}\left\{D_{z}^{\delta} f(z)\right\} \quad\left(0 \leq \delta<1 ; n \in \mathbb{N}_{0}\right) .
$$

Using Definitions 15, 16, and 17, we obtain

$$
\begin{gathered}
D_{z}^{\delta}\left\{z^{k}\right\}=\frac{\Gamma(k+1)}{\Gamma(k+1-\delta)} z^{k-\delta} \quad(k \in \mathbb{N} ; 0 \leq \delta<1), \\
D_{z}^{-\delta}\left\{z^{k}\right\}=\frac{\Gamma(k+1)}{\Gamma(k+1+\delta)} z^{k+\delta} \quad(k \in \mathbb{N} ; \delta>0),
\end{gathered}
$$

in terms of Gamma functions.

Theorem 18. Let the function $f(z)$ defined by (10) be in the class $T U_{m, n}(\beta, A, B)$. Then

$$
\begin{aligned}
& \left|D_{z}^{-\delta} f(z)\right| \\
& \begin{array}{l}
\geq \frac{|z|^{1+\delta}}{\Gamma(2+\delta)} \\
\quad \times\left(1-\frac{(A-B)}{(2+\delta)\left[(1+\beta(1+|B|))\left(2^{m-1}-2^{n-1}\right)+\left|B 2^{m-1}-A 2^{n-1}\right|\right]}|z|\right), \\
\left|D_{z}^{-\delta} f(z)\right| \quad(\delta>0 ; z \in U), \\
\leq \frac{|z|^{1+\delta}}{\Gamma(2+\delta)} \\
\quad \times\left(1+\frac{(A-B)}{(2+\delta)\left[(1+\beta(1+|B|))\left(2^{m-1}-2^{n-1}\right)+\left|B 2^{m-1}-A 2^{n-1}\right|\right]}|z|\right),
\end{array} \\
& \quad(\delta>0 ; z \in U) .
\end{aligned}
$$

The results are sharp.

Proof. Let

$$
\begin{aligned}
F(z) & =\Gamma(2+\delta) z^{-\delta} D_{z}^{-\delta} f(z) \\
& =z-\sum_{k=2}^{\infty} \frac{\Gamma(k+1) \Gamma(2+\delta)}{\Gamma(k+1+\delta)} a_{k} z^{k} \\
& =z-\sum_{k=2}^{\infty} \Lambda(k) a_{k} z^{k}
\end{aligned}
$$


where

$$
\Lambda(k)=\frac{\Gamma(k+1) \Gamma(2+\delta)}{\Gamma(k+1+\delta)}, \quad(k=2,3, \ldots) .
$$

Since $\Lambda(k)$ is a decreasing function of $k$, we can write

$$
0<\Lambda(k) \leq \Lambda(2)=\frac{2}{2+\delta} .
$$

Furthermore, in view of Theorem 4, we have

$$
\begin{aligned}
& {\left[(1+\beta(1+|B|))\left(2^{m}-2^{n}\right)+\left|B 2^{m}-A 2^{n}\right|\right] \sum_{k=2}^{\infty} a_{k}} \\
& \quad \leq \sum_{k=2}^{\infty}\left[(1+\beta(1+|B|))\left(k^{m}-k^{n}\right)+\left|B k^{m}-A k^{n}\right|\right] a_{k} \\
& \quad \leq A-B .
\end{aligned}
$$

Then

$$
\sum_{k=2}^{\infty} a_{k} \leq \frac{A-B}{\left[(1+\beta(1+|B|))\left(2^{m}-2^{n}\right)+\left|B 2^{m}-A 2^{n}\right|\right]} .
$$

Therefore, by using (85) and (87), we can see that

$$
\begin{aligned}
& |F(z)| \\
& \geq|z|-\Lambda(2)|z|^{2} \sum_{k=2}^{\infty} a_{k} \geq|z| \\
& \quad-\frac{(A-B)}{(2+\delta)\left[(1+\beta(1+|B|))\left(2^{m-1}-2^{n-1}\right)+\left|B 2^{m-1}-A 2^{n-1}\right|\right]}|z|^{2}
\end{aligned}
$$

and similarly

$$
\begin{aligned}
& |F(z)| \\
& \leq|z|+\Lambda(2)|z|^{2} \sum_{k=2}^{\infty} a_{k} \leq|z| \\
& \quad+\frac{(A-B)}{(2+\delta)\left[(1+\beta(1+|B|))\left(2^{m-1}-2^{n-1}\right)+\left|B 2^{m-1}-A 2^{n-1}\right|\right]}|z|^{2},
\end{aligned}
$$

which prove Theorem 18 .

Finally, the equalities are attained for the function $f(z)$ defined by

$$
\begin{aligned}
& D_{z}^{-\delta} f(z) \\
& =\frac{z^{1+\delta}}{\Gamma(2+\delta)} \\
& \quad \times\left(1-\frac{(A-B)}{(2+\delta)\left[(1+\beta(1+|B|))\left(2^{m-1}-2^{n-1}\right)+\left|B 2^{m-1}-A 2^{n-1}\right|\right]} z\right)
\end{aligned}
$$

or, equivalently, by $f(z)$ given by (27).

Then the results are sharp, and the proof of Theorem 18 is completed.
Corollary 19. Under the hypothesis of Theorem 20, $D_{z}^{-\delta} f(z)$ is included in a disk with its center at the origin and radius $R_{1}$ given by

$$
\begin{aligned}
R_{1} \\
=\frac{1}{\Gamma(2+\delta)} \\
\quad \times\left(1-\frac{(A-B)}{(2+\delta)\left[(1+\beta(1+|B|))\left(2^{m-1}-2^{n-1}\right)+\left|B 2^{m-1}-A 2^{n-1}\right|\right]}\right) .
\end{aligned}
$$

Theorem 20. Let the function $f(z)$ defined by (10) be in the class $T U_{m, n}(\beta, A, B)$. Then

$$
\left|D_{z}^{\delta} f(z)\right|
$$

$$
\begin{aligned}
\geq & \frac{|z|^{1-\delta}}{\Gamma(2-\delta)} \\
& \times\left(1-\frac{(A-B)}{(2-\delta)\left[(1+\beta(1+|B|))\left(2^{m-1}-2^{n-1}\right)+\left|B 2^{m-1}-A 2^{n-1}\right|\right]}|z|\right),
\end{aligned}
$$

$(0 \leq \delta<1 ; z \in U)$,

$$
\begin{aligned}
& \left|D_{z}^{\delta} f(z)\right| \\
& \leq \frac{|z|^{1-\delta}}{\Gamma(2-\delta)} \\
& \quad \times\left(1+\frac{(A-B)}{(2-\delta)\left[(1+\beta(1+|B|))\left(2^{m-1}-2^{n-1}\right)+\left|B 2^{m-1}-A 2^{n-1}\right|\right]}|z|\right),
\end{aligned}
$$

$(0 \leq \delta<1 ; z \in U)$

Each of these results is sharp.

Proof. Let

$$
\begin{aligned}
G(z) & =\Gamma(2-\delta) z^{\delta} D_{z}^{\delta} f(z) \\
& =z-\sum_{k=2}^{\infty} \frac{\Gamma(k) \Gamma(2-\delta)}{\Gamma(k+1-\delta)} k a_{k} z^{k}=z-\sum_{k=2}^{\infty} \Omega(k) k a_{k} z^{k}
\end{aligned}
$$

where

$$
\Omega(k)=\frac{\Gamma(k) \Gamma(2-\delta)}{\Gamma(k+1-\delta)} \quad(k=2,3, \ldots) .
$$

Since $\Omega(k)$ is a decreasing function of $k$, we can write

$$
0<\Omega(k) \leq \Omega(2)=\frac{1}{2-\delta} .
$$


Furthermore, in view of Theorem 4, we have

$$
\begin{aligned}
& {\left[(1+\beta(1+|B|))\left(2^{m-1}-2^{n-1}\right)+\left|B 2^{m-1}-A 2^{n-1}\right|\right] \sum_{k=2}^{\infty} k a_{k}} \\
& \quad \leq \sum_{k=2}^{\infty}\left[(1+\beta(1+|B|))\left(k^{m}-k^{n}\right)+\left|B k^{m}-A k^{n}\right|\right] a_{k} \\
& \quad \leq A-B .
\end{aligned}
$$

Then

$$
\begin{aligned}
& \sum_{k=2}^{\infty} k a_{k} \\
& \quad \leq \frac{A-B}{\left[(1+\beta(1+|B|))\left(2^{m-1}-2^{n-1}\right)+\left|B 2^{m-1}-A 2^{n-1}\right|\right]} .
\end{aligned}
$$

Therefore, by using (95) and (97), we can see that

$$
\begin{aligned}
& |G(z)| \\
& \geq|z|-\Omega(2)|z|^{2} \sum_{k=2}^{\infty} k a_{k} \geq|z| \\
& \quad-\frac{(A-B)}{(2-\delta)\left[(1+\beta(1+|B|))\left(2^{m-1}-2^{n-1}\right)+\left|B 2^{m-1}-A 2^{n-1}\right|\right]}|z|^{2}
\end{aligned}
$$

and similarly

$$
\begin{aligned}
& |G(z)| \\
& \leq|z|+\Omega(2)|z|^{2} \sum_{k=2}^{\infty} k a_{k} \leq|z| \\
& \quad+\frac{(A-B)}{(2-\delta)\left[(1+\beta(1+|B|))\left(2^{m-1}-2^{n-1}\right)+\left|B 2^{m-1}-A 2^{n-1}\right|\right]}|z|^{2},
\end{aligned}
$$

which together prove the two assertions of Theorem 20 .

Finally, the equalities are attained for the function $f(z)$ defined by

$$
\begin{aligned}
& D_{z}^{\delta} f(z) \\
& =\frac{z^{1-\delta}}{\Gamma(2-\delta)} \\
& \quad \times\left(1-\frac{(A-B)}{(2-\delta)\left[(1+\beta(1+|B|))\left(2^{m-1}-2^{n-1}\right)+\left|B 2^{m-1}-A 2^{n-1}\right|\right]} z\right)
\end{aligned}
$$

or, equivalently, by $f(z)$ given by (27).

Then the result is sharp, and the proof of Theorem 20 is completed.
Corollary 21. Under the hypothesis of Theorem 20, $D_{z}^{\delta} f(z)$ is included in a disk with its center at the origin and radius $R_{2}$ given by

$$
\begin{aligned}
R_{2} \\
=\frac{1}{\Gamma(2-\delta)} \\
\\
\quad \times\left(1-\frac{(A-B)}{(2-\delta)\left[(1+\beta(1+|B|))\left(2^{m-1}-2^{n-1}\right)+\left|B 2^{m-1}-A 2^{n-1}\right|\right]}\right) .
\end{aligned}
$$

\section{Acknowledgment}

The authors thank the referee for his valuable suggestions which led to improvement of this study.

\section{References}

[1] G. S. Salagean, "Subclasses of univalent functions," in Complex Analysis: Fifth Romanian-Finnish Seminar, vol. 1013 of Lecture Notes in Mathematics, pp. 362-372, Springer, Berlin, UK, 1983.

[2] S. S. Miller and P. T. Mocanu, Differential Subordinations: Theory and Applications, vol. 225 of Monographs and Textbooks in Pure and Applied Mathematics, Marcel Dekker, New York, NY, USA, 2000.

[3] S.-H. Li and H. Tang, "Certain new classes of analytic functions defined by using the Salagean operator," Bulletin of Mathematical Analysis and Applications, vol. 2, no. 4, pp. 62-75, 2010

[4] S. S. Eker and S. Owa, "Certain classes of analytic functions involving Salagean operator," Journal of Inequalities in Pure and Applied Mathematics, vol. 10, no. 1, pp. 12-22, 2009.

[5] S. Shams, S. R. Kulkarni, and J. M. Jahangiri, "On a class of univalent functions defined by Ruschweyh derivatives," Kyungpook Mathematical Journal, vol. 43, no. 4, pp. 579-585, 2003.

[6] S. Shams, S. R. Kulkarni, and J. M. Jahangiri, "Classes of uniformly starlike and convex functions," International Journal of Mathematics and Mathematical Sciences, no. 53, pp. 2959-2961, 2004.

[7] W. Janowski, "Some extremal problems for certain families of analytic functions," Annales Polonici Mathematici, vol. 28, pp. 648-658, 1973.

[8] K. S. Padmanabhan and M. S. Ganesan, "Convolutions of certain classes of univalent functions with negative coefficients," Indian Journal of Pure and Applied Mathematics, vol. 19, no. 9, pp. 880-889, 1988.

[9] T. Rosy and G. Murugusundaramoorthy, "Fractional calculus and their applications to certain subclass of uniformly convex functions," Far East Journal of Mathematical Sciences, vol. 15, no. 2, pp. 231-242, 2004.

[10] M. K. Aouf, "A subclass of uniformly convex functions with negative coefficients," Mathematica, vol. 52, no. 2, pp. 99-111, 2010.

[11] R. Bharati, R. Parvatham, and A. Swaminathan, "On subclasses of uniformly convex functions and corresponding class of starlike functions," Tamkang Journal of Mathematics, vol. 28, no. 1, pp. 17-32, 1997.

[12] H. Silverman, "Univalent functions with negative coefficients," Proceedings of the American Mathematical Society, vol. 51, pp. 109-116, 1975. 
[13] M. K. Aouf, R. M. El-Ashwah, A. A. M. Hassan, and A. H. Hassan, "On subordination results for certain new classes of analytic functions defined by using Salagean operator," Bulletin of Mathematical Analysis and Applications, vol. 4, no. 1, pp. 239-246, 2012.

[14] J. E. Littlewood, "On inequalities in the theory of functions," Proceedings of the London Mathematical Society, vol. 23, no. 1, pp. 481-519.

[15] H. M. Srivastava and M. K. Aouf, "A certain fractional derivative operator and its applications to a new class of analytic and multivalent functions with negative coefficients I," Journal of Mathematical Analysis and Applications, vol. 171, no. 1, pp. 1-13, 1992.

[16] H. M. Srivastava and M. K. Aouf, "A certain fractional derivative operator and its applications to a new class of analytic and multivalent functions with negative coefficients, II," Journal of Mathematical Analysis and Applications, vol. 192, no. 3, pp. 673-688, 1995.

[17] H. M. Srivastava and S. Owa, "Some characterization and distortion theorems involving fractional calculus, generalized hypergeometric functions, Hadamard products, linear operators, and certain subclasses of analytic functions," Nagoya Mathematical Journal, vol. 106, pp. 1-28, 1987.

[18] H. M. Srivastava and S. Owa, Univalent Functions, Fractional Calculus, and Their Applications, Halsted Press, Horwood Limited, Chichester, UK; John Wiley and Sons, New York, NY, USA, 1989. 


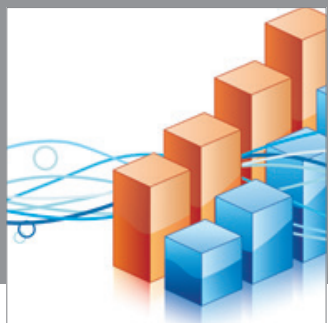

Advances in

Operations Research

mansans

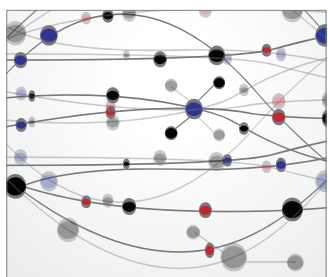

The Scientific World Journal
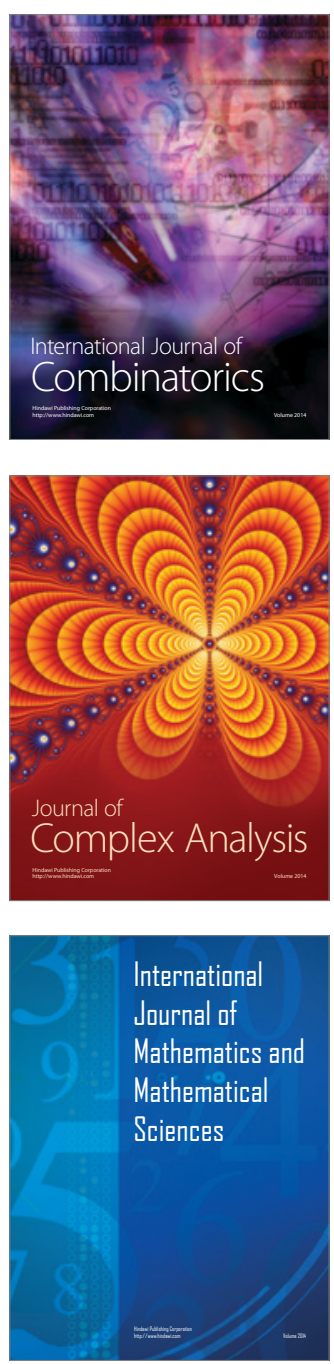
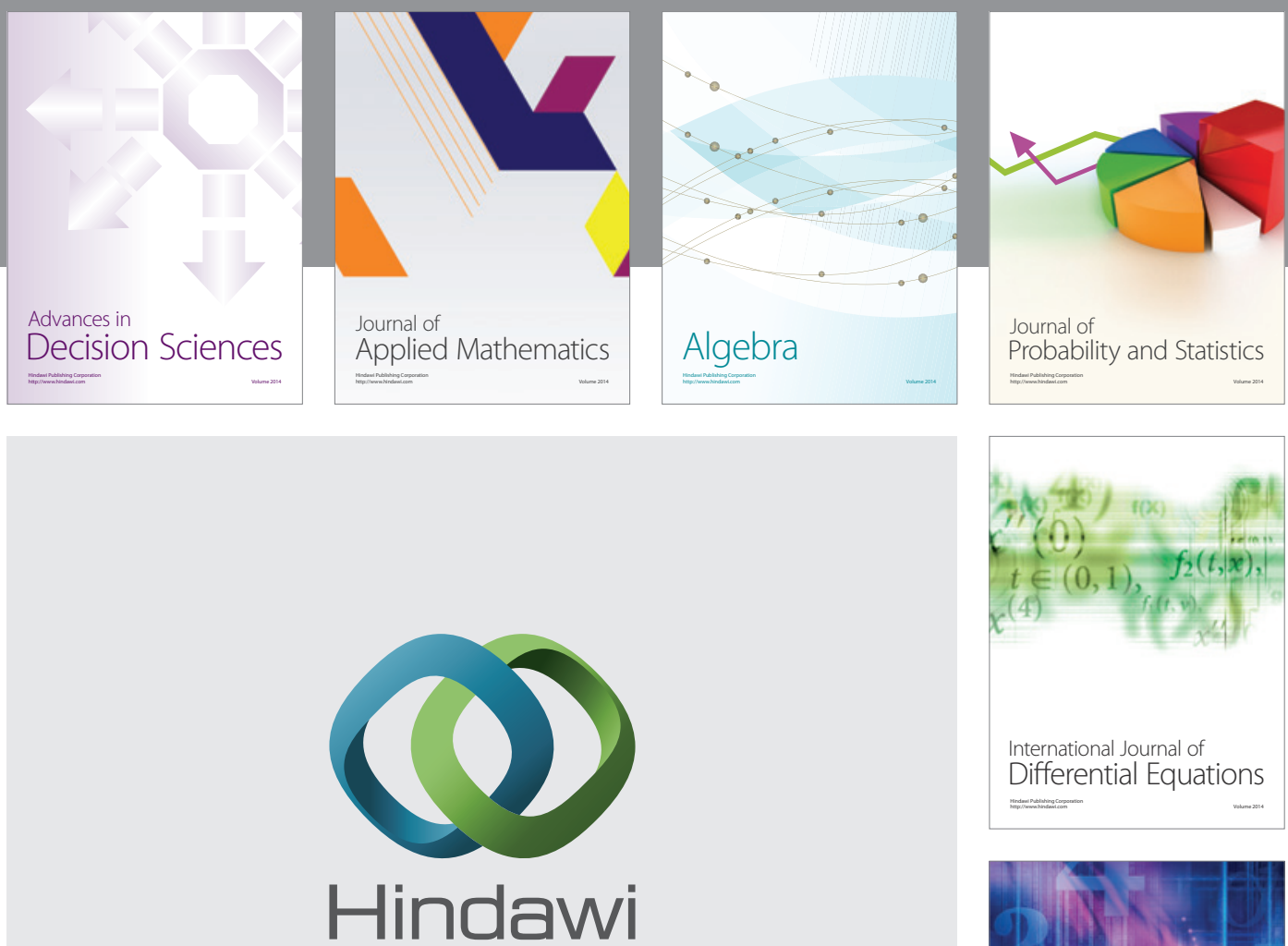

Submit your manuscripts at http://www.hindawi.com
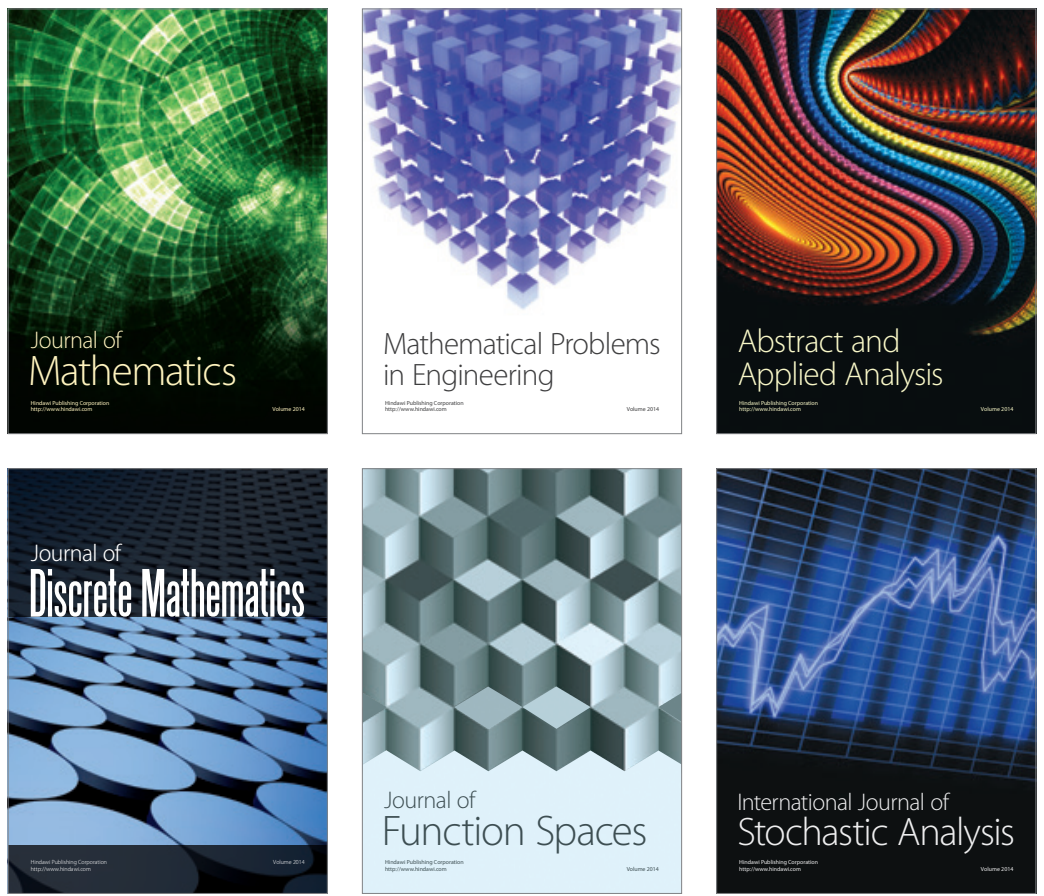

Journal of

Function Spaces

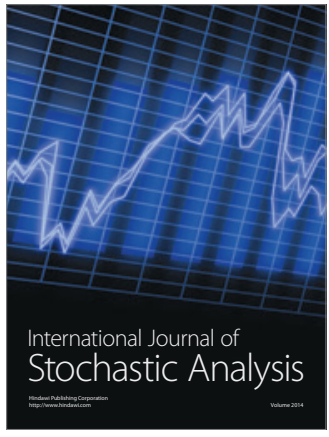

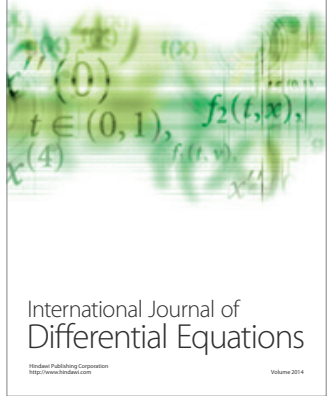
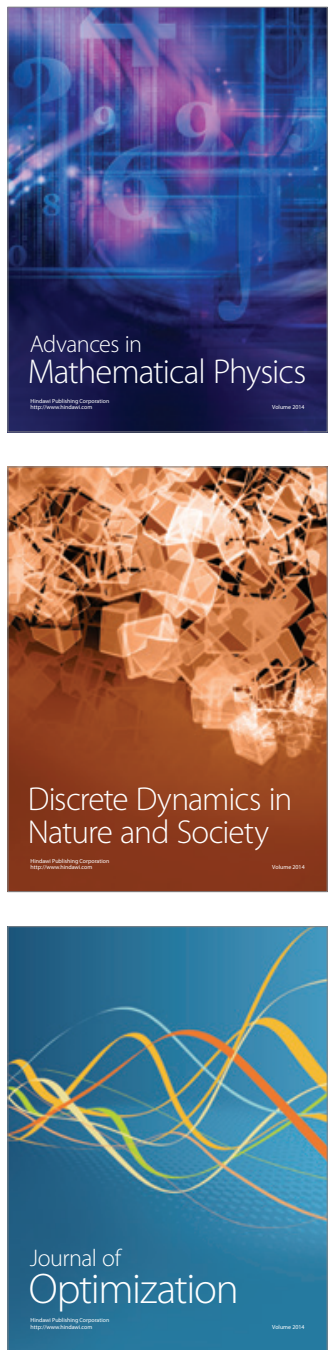